\title{
Polonium 210, Exposed
}

\author{
Mai H. Le, MD
}

University of California, San Francisco, Department of Laboratory Medicine

The death of a former KGB member, Alexander Litvinenko, from Polonium-210 (210Po) poisoning on November 23, 2006, has sparked a wave of fear and intrigue in this age of orange Homeland Security levels. Discovery of the radioactive heavy metal in Litvinenko's home, a sushi restaurant where he ate, and in the homes of several personal contacts has increased public anxiety. Concerns about the use of $210 \mathrm{Po}$ as a potential terrorist weapon have initiated an effort by the CDC and international health agencies to inform the public of the realities of $210 \mathrm{Po}$ and other radiological weapons.

In 1898 Marie and Pierre Curie, Nobel Laureates, discovered $210 \mathrm{Po}$, a naturally occurring radioactive heavy metal found in the soil and atmosphere at roughly one part per $10^{15}$, and it is also present at higher levels in tobacco smoke as well as a variety of industrial machines as an eliminator of static energy $[1,2]$. Following the emission of an alpha particle, two protons and two neutrons (such as a helium nucleus), the ${ }^{210}$ Po molecule decays into stable Lead $206(206 \mathrm{~Pb})$ with a half-life of approximately 138 days.

Alpha particles are the largest of the four types of radiation (alpha particle, beta particle, gamma ray, and neutron emission), and they are the least penetrating, incapable of passing through paper, clothing, or the keratinic layer of the epidermis. However, upon exposure to living cells, alpha particles are considered to be twenty times as damaging as X-ray or gamma radiation [3]. The multiple organ damage associated with its toxicity, as seen in the case of Mr. Litvinenko, results when ${ }^{210} \mathrm{Po}$ is ingested, inhaled, or introduced through disrupted skin.

Upon ingestion, between $50 \%$ to $90 \%$ of ${ }^{210} \mathrm{Po}$ is excreted in the feces [2]. The remaining ${ }^{210} \mathrm{Po}$ is distributed throughout the body with the highest concentrations in the plasma, spleen, kidneys, and liver [4]. Because the largest pool of ${ }^{210} \mathrm{Po}$ is initially found in the bloodstream, vascular endothelial cells are a primary target for the short-range effects of alpha particles. The radiation also depletes tissues of stem cell reserves. The result of an acute, high dose exposure is Acute Radiation Syndrome (ARS).

A fact sheet is available to physicians on the CDC website even though the information is aimed more towards beta particle and gamma radiation exposures. According to the fact sheet, the conditions necessary to cause ARS are:

(1) A large radiation dosage (greater than 70 rads). To provide some perspective, the radiation exposure to a patient during a head CT is $\sim 2$ rads. For a two-view chest X-ray, the radiation exposure is 0.006 rads.

(2) An external radiation source (such as a source of radiation outside the patient's body). Obviously, this does not apply to radiation poisoning secondary to alpha emitters since these particles cannot penetrate the epidermis.

(3) The radiation must be penetrating (able to reach internal organs). Again, this does not apply to alpha emitters. Ingestion or inhalation is necessary for alpha emitters to affect internal organs.

(4) Full body exposure.

(5) A short time of delivery (delivery of full dosage within minutes) [5].

Cell damage by alpha particles appears to occur both by direct damage as well as via a bystander effect. Cell damage by direct exposure is primarily due to single or double-strand DNA breaks, resulting in both mutagenic and cytotoxic effects. In a series of studies evaluating the effects of environmentally-relevant doses of alpha particles, Kadhim et al. found that exposure on haematopoeitic stem cells resulted in chromosomal instability transmissible to subsequent culture generations [6]. Alpha particles also appear to increase the frequency of sister chromatid exchanges [7]. Furthermore, DNA damage is not limited to cells directly hit by alpha particles. Experimental evidence suggests

Notes: There was no outside funding of any kind used in this study. Cooresponding Author: Mai H. Le, MD Email: mai.le@ucsfmedcrt.org 
that DNA damage can also affect the unirradiated neighbors via cell signaling pathways, the so-called 'bystander effect' $[8,9]$. As expected with substances that cause DNA breaks, the most profound affect of alpha particles is on rapidly dividing cells, including those of the GI tract, bone marrow, skin, hair, and nails.

The signs and symptoms of ${ }^{210} \mathrm{Po}$ poisoning are that of ARS, and the clinical severity is proportional to the exposure dosage. The three classic syndromes associated with ARS are (1) Bone Marrow Syndrome, (2) Gastrointestinal Syndrome, and (3) Cardiovascular/ Central Nervous System Syndrome. The full syndromes typically occur at 70-1000 rads, 1000-10,000 rads, and >5,000 rads, respectively; although, manifestations may occur at lower radiation levels.

ARS occurs in four phases that may include one or more of the three classic syndromes. The time-to-onset as well as the length of each stage are highly variable and, as one would expect, dependent upon radiation dosage. Overt symptoms of the first stage (Prodromal Stage) include nausea, vomiting, diarrhea, anorexia, fever, extreme nervousness and/or confusion, loss of consciousness, and a burning sensation of the skin. Destruction of haematopoeitic precursors in the bone marrow also occurs in the Prodromal Stage. This phase, which lasts for minutes to days, is then followed by a period of apparent clinical improvement (i.e. 'Latent phase') lasting several hours to 6 weeks. In the Latent Phase, the patient appears well despite continued bone marrow suppression and death of the GI epithelium. In those who continue to decline, the next stage ('Manifest Illness Phase') is characterized by extreme immunosuppression and mucosal bleeding secondary to bone marrow failure and pancytopenia. Severe electrolyte imbalances from diarrhea and dehydration can also be seen. Death often occurs within 2-3 weeks of the onset of the Manifest Illness Phase.

The diagnosis of ARS secondary to ${ }^{210}$ Po is difficult for a number of reasons. First and foremost, the clinical index of suspicion is exceedingly low. Litvinenko may have been the only person in history since its discovery to be deliberately poisoned and killed by ${ }^{210}$ Po. Only a handful of other deaths are thought to be due to 210Po exposure, and none of them from ARS. Irene Joliot-Curie, daughter of Marie and Pierre Curie, was the first person to die of known ${ }^{210}$ Po exposure. After an accidental explosion of sealed 210Po, she died in Paris 10 years later of leukemia [10]. The deaths of several Israeli radiation scientists from cancer are also postulated to be secondary to ${ }^{210} \mathrm{Po}[11]$.

The clinical features at any given point of ARS are rather nonspecific and could easily be mistaken for a variety of other, more common diagnoses. In a patient who is acutely ill, but with no known history of radiation exposure, the presenting symptoms (primarily malaise, nausea, and vomiting) would be most suggestive of an infectious etiology. The variable haematologic, gastrointestinal, and cardiovascular and/or central nervous system manifestations could encompass anything, from acute HIV syndrome to metastatic malignancy. The diagnosis might only become clear in hindsight after the manifestations of ARS have taken their full course, and only palliation can be offered.
Furthermore, standard techniques cannot detect 210Po in the body because it does not penetrate the skin from the outside in, or the inside out. Specific detection of alpha particles from a patient's urine or feces is necessary. Fortunately, the biological half-life of ${ }^{210} \mathrm{Po}$ (30-50 days) offers some opportunity to screen samples ex post facto.

The mean lethal dose (LD50) for an acute radiation exposure is about $4 \mathrm{~Sv}$, which is roughly equivalent to ingesting $50 \mathrm{ng}$ or inhaling $10 \mathrm{ng}$ of ${ }^{210} \mathrm{Po}$ [12]. Since naturally occurring ${ }^{210} \mathrm{Po}$ is exceedingly rare, the amount needed to poison must be manufactured by bombarding natural bismuth with neutrons. This process requires the high-neutron fluxes of a nuclear reactor, and those seeking 210Po must have the political resources to infiltrate and access nuclear facilities. The death of Mr. Litvinenko's by 210 Po exposure lends itself to the lore of espionage and political conspiracy.

It is plausible that terrorist organizations are adding radioactive substances to their arsenal. Radiological Dispersion Devices such as dirty bombs (conventional explosives containing radioactive material) have been used on a number of occasions to kill and injure those within the immediate vicinity, contaminate land and buildings, and induce widespread fear [13]. The death of Litvinenko from ARS only increased fear of radiation exposure.

However, ${ }^{210}$ Po would be an unlikely candidate for largescale radiation poisoning for several reasons. First, there are other sources of radiation that that are easier to obtain. ${ }^{210} \mathrm{Po}$ is extremely rare and obtaining adequate amounts for largescale destruction requires significant facilities and resources. Other radioactive heavy metals are more readily available in medical, research, and industrial settings. For example, Cesium137 (gamma emission) is commonly found at cancer treatment centers. Several events in the past 20 years (including an incident in Greensboro, North Carolina (March 1998) in which 19 tubes of cesium were stolen) indicate that it is not too difficult to obtain. Secondly, ${ }^{210}$ Po requires access to a mucosal surface or wound in order to cause ARS. It would not be very effective in a dirty bomb because, aside from those in the immediate proximity to the explosion who are either wounded or inhale large quantities of radioactive dust, the alpha particles would not have much penetrance. Therefore, large-scale poisoning is unlikely to occur with 210 Po. Terrorists are more likely to use devices that emit beta particles or gamma radiation, both of which penetrate the skin. Cesium-137, Plutonium-238, and Strontium-90 are more practical than $210 \mathrm{Po}$. And, the most probable delivery system would be a dirty bomb rather than direct poisoning.

Although the chance of widespread 210 Po poisoning is essentially zero, the Litvinenko saga has served to remind the medical community of the clinical features of Acute Radiation Syndrome. It is important for medical toxicologists to recognize ARS in order to provide the most appropriate care to the individual as well as to provide support in the event of large-scale uses of radioactive weapons.

The author has no potential financial conflicts of interest to report. 


\section{REFERENCES}

1. Kilthau GF. Cancer risk in relation to radioactivity in tobacco. Radiol Technol. 1996;67(3):217-222.

2. Argonne National Laboratory, E, Polonium. Human Health Fact Sheet, 2005.

3. IRCP, Recommendations of the International Commision on Radiological Protection, Ann. IRCP, 1991;No. 3.

4. Leggett RW, Eckerman KF. A systemic biokinetic model for polonium. Sci Total Environ. 2001;275(1-3):109-125.

5. Acute Radiation Syndrome Fact Sheet for Physicians. Centers for Disease Control and Prevention.

6. Kadhim MA, et al. Transmission of chromosomal instability after plutonium alpha-particle irradiation. Nature.

1992;355(6362):738-740.

7. Nagasawa H, Little JB. Induction of sister chromatid exchanges by extremely low doses of alpha-particles. Cancer Res. 1992;52(22):6394-6396.
8. Zhou $\mathrm{H}$, et al. Induction of a bystander mutagenic effect of alpha particles in mammalian cells. Proc Natl Acad Sci U S A. 2000;97(5):2099-2104.

9. Zhou $\mathrm{H}$, et al. Mechanism of radiation-induced bystander effect: role of the cyclooxygenase- 2 signaling pathway. Proc Natl Acad Sci U S A. 2005;102(41): 14641-14646.

10. The Manhattan Project Heritage Preservation Association, I., Irene Joliot-Curie.

11. Karpin M. The Bomb in the Basement: How Israel Went Nuclear and What That Means for the World. Simon \& Schuster, Inc., January 2006.

12. Strom DJ. Health Impacts from Acute Radiation Exposure, P.N.N. Laboratory, Editor. 2003;U.S. Department of Energy.

13. Krock LaD. Rebecca, Dirty Bomb: Chronology of Events. NOVA Science Programming on Air and Online. 